\title{
EDUCAÇÃO EM SAÚDE PARA A QUALIDADE DE VIDA DE USUÁRIOS DE SUBSTÂNCIAS PSICOATIVAS
}

\section{HEALTH EDUCATION FOR THE QUALITY OF LIFE OF USERS OF PSYCHOACTIVE SUBSTANCES}

\section{EDUCACIÓN EN SALUD PARA LA CALIDAD DE VIDA DE LOS USUARIOS DE SUSTANCIAS PSICOACTIVAS}

\author{
Claudia Cristina Rolim da Silva ${ }^{1}$ \\ Célio Fernando de Sousa Rodrigues ${ }^{2}$
}

Como citar este artigo: Silva CCR, Rodrigues CFS. Educação em saúde para a qualidade de vida de usuários de substâncias psicoativas. Rev baiana enferm. 2021;35:e39041.

Objetivo: comparar os impactos de duas estratégias educativas em saúde na qualidade de vida de pessoas com transtornos por uso de substâncias psicoativas. Método: estudo descritivo, analítico e experimental realizado com 40 usuários de um Centro de Atenção Psicossocial Álcool e Outras Drogas. Foi aplicado um questionário para comparação da adesão ao tratamento e os problemas relatados pelos usuários em tratamento, antes e após a aplicação de duas estratégias educativas. As variáveis estudadas foram a adesão em relação ao tratamento e a frequência dos problemas nos âmbitos financeiro, social e de saúde. Resultados: no grupo com intervenções dialógicas, houve maior pontuação na adesão e melhor ganho no aspecto econômico, familiar e social em comparação ao grupo com estratégia tradicional. Não houve diferenças no âmbito da saúde. Conclusão: a estratégia educativa dialógica demonstrou ser mais efetiva na resolução de alguns problemas relatados por usuários de substâncias psicoativas.

Descritores: Educação em Saúde. Qualidade de Vida. Saúde Mental. Transtornos por Uso de Substâncias Psicoativas. Enfermagem.

Objective: to compare the impacts of two health educational strategies on the quality of life of people with psychoactive substance use disorders. Method: descriptive, analytical and experimental study conducted with 40 users of a Psychosocial Care Center for Alcohol and Other Drugs. A questionnaire was applied to compare treatment adherence and the problems reported by users undergoing treatment, before and after the application of two educational strategies. The variables studied were treatment adherence and frequency of problems in the financial, social and health spheres. Results: in the group with dialogical interventions, there was a higher score on the participation and better gain in the economic, family and social aspects compared to the group with traditional strategy. There were no differences in health. Conclusion: the dialogical educational strategy proved to be more effective in solving some problems reported by users of psychoactive substances.

Descriptors: Health Education. Quality of Life. Mental Health. Psychoactive Substance Use Disorders. Nursing.

Objetivo: comparar los impactos de dos estrategias educativas en la salud en la calidad de vida de las personas con trastornos del consumo de sustancias psicoactivas. Método: estudio descriptivo, analítico y experimental realizado con 40 usuarios de un Centro de Atención Psicosocial en Alcohol y Otras Drogas. Se aplicó un cuestionario para comparar el cumplimiento del tratamiento y los problemas reportados por los usuarios sometidos a tratamiento, antes

\footnotetext{
Enfermeira. Mestre em Ensino na Saúde e Tecnologia. Enfermeira efetiva da Estratégia de Saúde da Família da Prefeitura Municipal de Arapiraca. Arapiraca, Alagoas, Brasil. clarolimsilva@gmail.com. http://orcid.org/0000-0002-7019-2648.

Médico. Doutor em Morfologia. Professor da Universidade Estadual de Ciências da Saúde de Alagoas e da Universidade Federal de Alagoas. Maceió, Alagoas, Brasil. http://orcid.org/0000-0002-1361-8139.
} 
y después de la aplicación de dos estrategias educativas. Las variables estudiadas fueron la adhesión al tratamiento y la frecuencia de los problemas en los ámbitos financiero, social y de salud. Resultados: en el grupo con intervenciones con diálogos, bubo una puntuación más alta en la participación y una mejor ganancia en los aspectos económicos, familiares y sociales en comparación con el grupo con la estrategia tradicional. No bubo diferencias en la salud. Conclusión: la estrategia educativa con diálogos demostró ser más eficaz en la resolución de algunos problemas reportados por los usuarios de sustancias psicoactivas.

Descriptores: Educación para la Salud. Calidad de Vida. Salud Mental. Trastornos Psicoactivos por Consumo de Sustancias. Enfermería.

\section{Introdução}

A medição da qualidade de vida (QV) somente pode ser efetivada se a realidade dos indivíduos puder ser percebida de modo subjetivo e multidimensional, isto é, biopsicossocial ${ }^{(1)}$. Em relação à saúde, essa medição caracteriza-se pela impressão de cada pessoa, por meio de autorrelatos, quanto à capacidade de executar funções e ao desenvolvimento de habilidades em face das consequências patológicas instaladas ${ }^{(2)}$.

O uso de substâncias psicoativas ocasiona transtornos que afetam de modo negativo $\mathrm{e}$ direto e progridem ao longo da vida de modo desordenado. A prioridade pelo consumo gera comportamentos manipuladores e ilícitos, apesar da presença de problemas de ordens financeira, social e familiar ${ }^{(3-4)}$. No processo destrutivo que se instala, em algum momento, o sofrimento por sentir-se sozinho com todas as sequelas, desperta o indivíduo para a mudança de vida e, consequentemente, para a busca de soluções. Assim, a procura por ajuda de profissionais ${ }^{(5)}$ especializados em tratamento da dependência química passa a ser a única alternativa para a superação.

A educação em saúde é uma das principais ferramentas terapêuticas utilizadas em Centros de Atenção Psicossocial. Além disso, para o usuário, pode gerar oportunidades, promover a cidadania, a democratização do direito do saber e o empoderamento de pessoas e coletivos, pois possibilita não somente o conhecimento do processo saúde e doença ${ }^{(6)}$, como também pode favorecer a mudança de atitude e a melhoria da QV. Ações educativas estão entre as atribuições e responsabilidades do enfermeiro, que deve executá-las de tal modo que considere as reais necessidades sociais e coletivas. Desse modo, poderá promover estilos de vida saudáveis e tornar-se agente da transformação social ${ }^{(7)}$.

Cabe salientar que existem diferentes modos de se realizar a educação em saúde. Dentre esses, destacou-se, para fins deste estudo, dois modelos: tradicional, cuja transmissão do conhecimento, por ser verticalizada, com pouco ou quase nenhum diálogo, não promove a reflexão dos participantes; e o modelo educativo dialógico, que propõe diálogo e reflexão dos participantes com base em sua realidade, promovendo a construção do conhecimento de modo horizontal. Importa esclarecer que o modelo educativo utilizado interfere não só no planejamento como também na ação a ser realizada e também na estratégia escolhida para a aplicação da proposta educativa $^{(8-9)}$.

No caso dos usuários de substâncias psicoativas, é importante verificar se o tipo de estratégia educativa utilizada, mediante aplicação de ações de baixo custo e executadas cotidianamente por profissionais de saúde, pode melhorar a adesão ao tratamento e produzir melhorias na QV desses indivíduos. Desse modo, com base nos modelos destacados para este estudo, estabeleceu-se como estratégias educativas em saúde a tradicional e a dialógica.

Para clarificar o caminho da investigação e compreender a problemática em estudo, formulou-se a seguinte questão de pesquisa: Qual o impacto de duas estratégias educativas em saúde na Qualidade de Vida de pessoas com transtornos por uso de substâncias psicoativas? 
A resposta a esta questão poderá contribuir para reflexões e para a tomada de decisão de profissionais de saúde mental, bem como em outros espaços em que sejam aplicadas ações educativas em saúde e, assim, consiga-se melhorar a QV das pessoas e de seus coletivos.

O objetivo deste estudo é comparar os impactos de duas estratégias educativas em saúde na QV de pessoas com transtornos por uso de substâncias psicoativas.

\section{Método}

Trata-se de um estudo descritivo, analítico e experimental com grupo controle não equivalente. Para identificação dos resultados de cada intervenção em ambos os grupos, nos aspectos relacionados à saúde, ao financeiro e ao social, foi aplicado pré-teste e executadas intervenções individuais e em grupo, seguindo o modelo tradicional, no grupo controle - que foi denominado Grupo Tradicional (GT) - e também orientadas pelo modelo dialógico, no grupo experimental que recebeu o nome de Grupo Dialógico (GD).

Este estudo é um recorte da dissertação intitulada "Comparativo de duas Estratégias Educativas em Saúde e suas Relações nas Ações do Autocuidado de Usuários de Álcool e Outras Drogas". Foi executado com usuários de um Centro de Atenção Psicossocial Álcool e Outras Drogas (CAPS AD), em um município do estado de Alagoas. O recrutamento foi realizado por conveniência, com pessoas que se encontravam em tratamento, tendo como responsável o profissional Enfermeiro.

Os critérios de inclusão foram: pessoa do sexo masculino dependente de substâncias psicoativas com indicação de tratamento diário, três vezes na semana, duas vezes ou pontualmente no grupo de Autocuidado; ser cadastrada na instituição. No momento da entrevista, no pré e pós-teste, deveriam estar lúcidas, orientadas no tempo e no espaço, maiores de idade, e concordassem em participar do acompanhamento. Este ocorreria por meio de intervenções individuais e entrevistas no período de três meses. Foram excluídas pessoas que não participaram de nenhum grupo educativo, com cognição prejudicada no momento da entrevista de avaliação, do sexo feminino ou expressassem desejo de exclusão do estudo.

Deste modo, a amostra foi composta por 40 participantes do sexo masculino, divididos em grupo controle e grupo experimental. Não houve participantes do sexo feminino, por não se encontrarem dentro dos critérios de inclusão no período pesquisado.

A alocação em cada um dos dois grupos foi conseguida após a aplicação do pré-teste, tomando-se por base um instrumento de avaliação do autocuidado Agency-ASA-ESCALE (ASA-A).

Com a finalidade de buscar semelhanças entre os grupos - Grupo controle e Grupo experimental -, os participantes foram alocados de 1:1, com base na classificação do autocuidado obtida em outra pesquisa ${ }^{(10)}$.

Para avaliação do tipo de droga e da percepção dos problemas vivenciados pelos participantes em relação à saúde e aos aspectos sociais e financeiros, foi utilizado um questionário construído com base em três instrumentos validados no Brasil ${ }^{(11)}$. O questionário foi aplicado no pré-teste e no final do primeiro, do segundo e do terceiro mês, por telefone ou presencial, a critério do participante.

Os participantes foram convidados individualmente pela pesquisadora principal e, após a assinatura do Termo de Consentimento Livre e Esclarecido (TCLE), foi aplicado o pré-teste. Em seguida, foram incluídos nos dois grupos, para o início das intervenções em grupo e individuais. O referencial teórico utilizado para a execução dos grupos - tradicional e dialógico - foi determinado de acordo com as diferenças entre o modelo educativo tradicional, com uma estratégia bancária, verticalizada, e o modelo dialógico, pautado na construção coletiva do conhecimento baseada na realidade dos participantes ${ }^{(9)}$.

No período de outubro de 2018 a março de 2019, a cada grupo (GT e GD) foram aplicadas 14 intervenções.

As variáveis estudadas foram: adesão dos participantes e resolução dos problemas vivenciados. Nesta última foi observada a frequência 
dos problemas autorrelatados em relação aos aspectos financeiros, sociais e de saúde. Os registros foram tabelados e armazenados no programa Excel, de modo que os dados qualitativos foram apresentados na forma de quadro e frequência.

A adesão dos participantes foi verificada com base em dois ângulos: o primeiro, conforme o cálculo elaborado pela fórmula " $\mathrm{n}$ - de exposições x no de participantes", em que se identificaram aqueles que se expuseram à metodologia de cada intervenção, gerando o valor total analisado; o segundo, foi contabilizado pela quantidade da frequência de exposições. Os critérios deste estudo foram: frequência (F) menor que 4 exposições = adesão baixa ou insuficiente; frequência entre 5 e 6 exposições = adesão moderada; e frequência maior ou igual a 7 exposições = adesão boa ou ótima.

Esta pesquisa foi realizada após aprovação pelo Comitê de Ética em Pesquisa (CEP) da Universidade Estadual de Ciências da Saúde de Alagoas, sob Parecer no 2.768.354 e Certificado de Apresentação de Apreciação Ética (CAAE):
89195918.6.0000.5011, conforme preconiza a Resolução n⿳0 466, de 12 de dezembro de 2012, do Conselho Nacional de Saúde (CNS), que regulamenta as diretrizes e normas de pesquisas envolvendo seres humanos.

\section{Resultados}

Os resultados da pesquisa são apresentados em duas seções que abordam a adesão dos participantes aos dois grupos - Grupo Tradicional (GT) e Grupo Dialógico (GD) - e a resolução dos problemas (financeiros, de saúde e familiares relatados) vivenciados.

\section{Adesão dos participantes}

Os dados apontaram que houve maior adesão no grupo com intervenções dialógicas (97 pontos) em relação ao grupo com exposição tradicional (84 pontos), diferença de 13 pontos. A frequência nesses grupos é apresentada na Tabela 1.

Tabela 1 - Frequência dos participantes nos grupos tradicional e dialógico. Arapiraca, Alagoas, Brasil - 2019. $(N=40)$

\begin{tabular}{|c|c|c|c|c|}
\hline $\begin{array}{l}\text { Frequência } \\
\text { da exposição }\end{array}$ & $\begin{array}{c}\text { Grupo Tradicional } \\
\text { (no de } \\
\text { participantes) }\end{array}$ & $\begin{array}{c}\text { Pontuação } \\
\text { Grupo Tradicional } \\
\text { (GT) }\end{array}$ & $\begin{array}{c}\text { Grupo Dialógico } \\
\text { (no de } \\
\text { participantes) }\end{array}$ & $\begin{array}{c}\text { Pontuação } \\
\text { Grupo Dialógico } \\
\text { (GD) }\end{array}$ \\
\hline 1 & 3 & 3 & 2 & 2 \\
\hline 2 & 3 & 6 & 3 & 6 \\
\hline 3 & 5 & 15 & 2 & 6 \\
\hline 4 & 2 & 8 & 4 & 16 \\
\hline 5 & 1 & 5 & 2 & 10 \\
\hline 6 & 1 & 6 & - & - \\
\hline 7 & 2 & 14 & 1 & 7 \\
\hline 8 & - & - & 5 & 40 \\
\hline 9 & 3 & 27 & - & - \\
\hline 10 & - & - & 1 & 10 \\
\hline Total & $20 / 100$ & $84 / 100$ & $20 / 100$ & $97 / 100$ \\
\hline
\end{tabular}

Fonte: Elaboração própria.

Notas: 1 Frequência da exposição:

Adesão baixa ou insuficiente: frequência (F) menor que 4 exposições.

Adesão moderada: frequência (F) entre 5 e 6 exposições;

Adesão boa ou ótima: frequência (F) maior ou igual a 7 exposições.

2 Sinal convencional utilizado:

- Dado numérico igual a zero não resultante de arredondamento.

Em relação à frequência de participação, de acordo com os parâmetros metodológicos desta pesquisa, em ambos os grupos (GT e GD), verificou-se baixa adesão. Isso pode ser observado na Tabela 1, em que 75\% dos usuários do GT e 55\% do GD obtiveram frequência baixa, de 
até quatro exposições. Ambos os grupos obtiveram 10\% de adesão moderada.

Entretanto, identificou-se que houve melhor frequência, ainda que discreta, dos participantes que se expuseram à estratégia dialógica, classificada como "boa" no GD (35\%), em comparação ao GT (25\%). A estratégia dialógica favoreceu maior envolvimento dos usuários e maior frequência em relação ao grupo no qual foi aplicada a estratégia tradicional.

Apesar da menor adesão, os participantes do GT apresentaram interesse nas temáticas trazidas pelos facilitadores, especialmente quando possuíam relação com doenças que tinham associação com seu histórico, isto é, quando essas atividades conseguiam aproximar-se mais da sua realidade. Embora esse comportamento tenha ocorrido de modo singular e esporádico, o fato de estarem em grupo favoreceu a maior abertura do participante diante das orientações fornecidas no momento da intervenção.

\section{Resolução dos problemas vivenciados}

A despeito de haver atividades de educação, observou-se, em ambos os grupos, que não houve diminuição na frequência dos problemas e das situações vivenciadas em relação à saúde, ao passo que se verificaram diferenças em relação aos problemas familiares e aos aspectos econômicos entre os grupos GT e GD, como expressam os Gráficos 1 e 2, respectivamente.

Gráfico 1 - Tipos de problemas financeiros, de saúde e familiares relatados e vivenciados pelos participantes do Grupo Tradicional. Arapiraca, Alagoas, Brasil - 2019. (N=20)

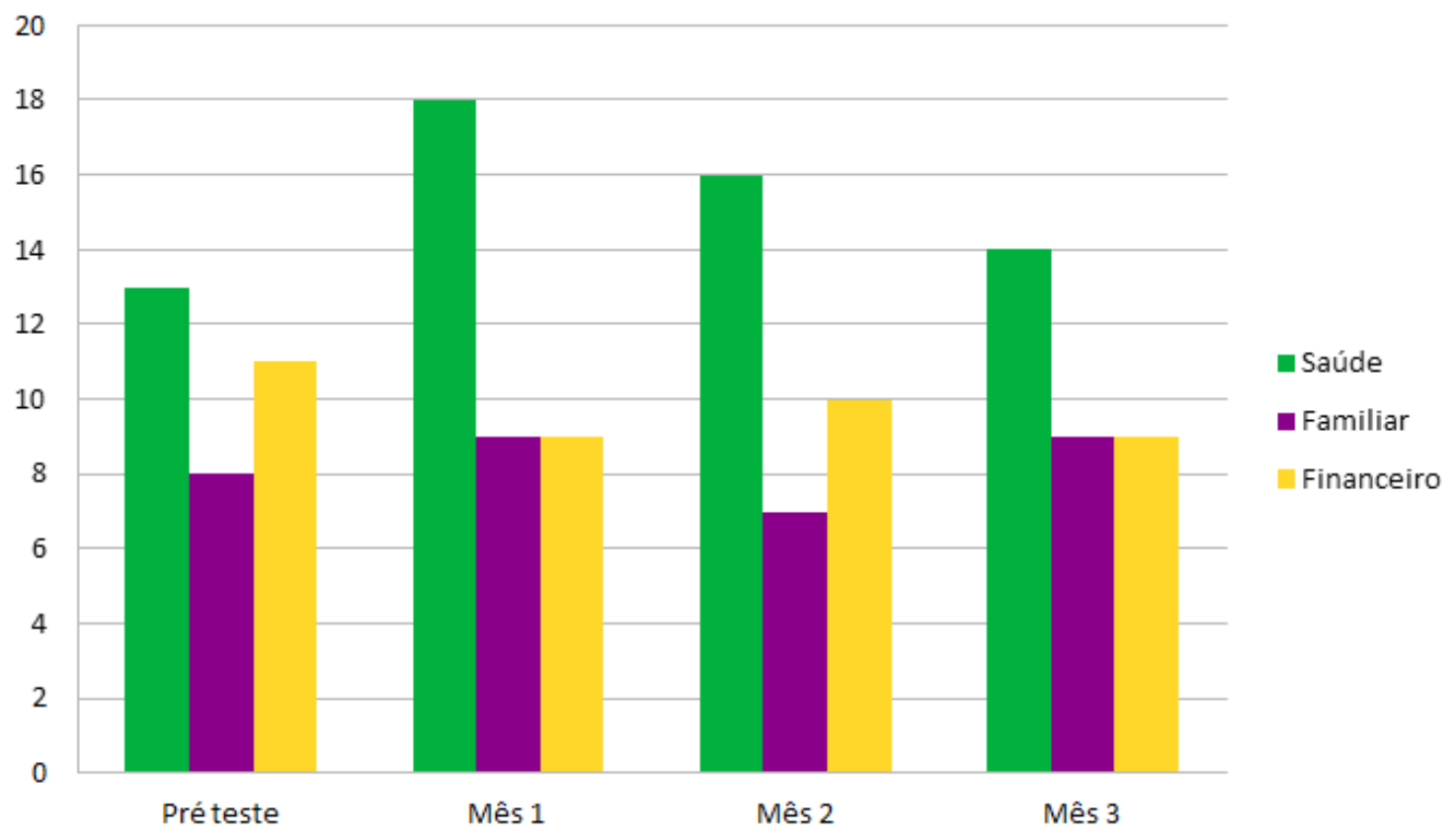

Fonte: Elaboração própria 
Gráfico 2 - Tipos de problemas financeiros, de saúde e familiares relatados e vivenciados pelos participantes do Grupo Dialógico. Arapiraca, Alagoas, Brasil - 2019. (N=20)

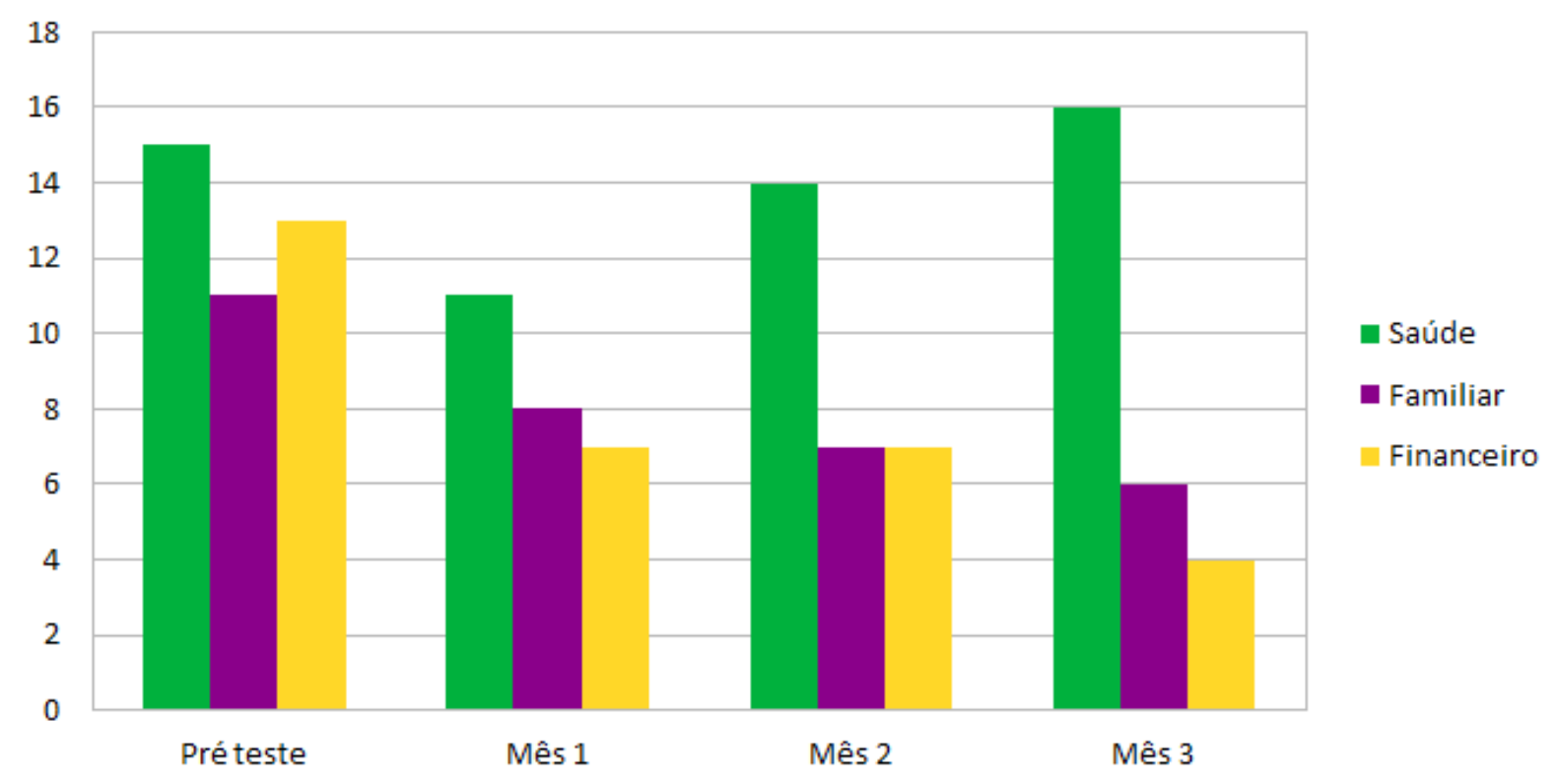

Fonte: Elaboração própria.

No tocante aos aspectos que envolvem o contexto de saúde, houve aumento de 1 ponto em relação ao pré-teste tanto no GT quanto no GD. Isto é, em ambos os grupos não foi observada melhora nos aspectos que envolveram a saúde dos participantes, independentemente das intervenções educativas.
Ainda em relação à saúde, durante o acompanhamento, os tipos de complicações identificadas e relatadas pelos participantes encontram-se descritos no Quadro 1.

Quadro 1 - Complicações de saúde referidas pelos participantes do Grupo Tradicional e do Grupo Dialógico. Arapiraca, Alagoas, Brasil - 2019. (N=40)

\begin{tabular}{|l|l|l|}
\hline $\begin{array}{l}\text { Tipos de } \\
\text { Complicações }\end{array}$ & \multicolumn{1}{|c|}{ Grupo Tradicional (GT) } & \multicolumn{1}{c|}{ Grupo Dialógico (GD) } \\
\hline Psiquiátricas & $\begin{array}{l}\text { Nervosismo, ansiedade, tristeza, } \\
\text { depressão, insônia }\end{array}$ & $\begin{array}{l}\text { Agressividade, nervosismo, ansiedade, } \\
\text { depressão, tentativa de suicídio }\end{array}$ \\
\hline Neurológicas & Alucinações, transtornos convulsivos & Alucinações, transtornos convulsivos \\
\hline Cardiovasculares & $\begin{array}{l}\text { Diabetes, hipertensão, edema, dor } \\
\text { precordial, hipotensão }\end{array}$ & Edema \\
\hline Gastrointestinais & Gastrite & Diarreia, vômitos, azia, anemia \\
\hline Traumas & Fratura, cirurgia & Fratura, ferimento por perfurocortante \\
\hline Infecções parasitárias & Hanseníase, esquistossomose & - \\
\hline Esqueléticas & Lombalgia & Lombalgia \\
\hline Outras complicações & $\begin{array}{l}\text { Tremores, inapetência, debilidade } \\
\text { física, fraqueza, cefaleia }\end{array}$ & $\begin{array}{l}\text { Tremores, dor, disúria, febre, } \\
\text { debilidade, fraqueza, exantema, } \\
\text { asma }\end{array}$ \\
\hline
\end{tabular}

Fonte: Elaboração própria.

Durante a aplicação da estratégia dialógica, os participantes demonstraram maior interesse em discutir doenças psiquiátricas, com ênfase na depressão e no suicídio, o que possibilitou identificar pessoas com estado depressivo e risco elevado. 
No GT percebeu-se maior envolvimento em relação às doenças crônicas em detrimento das questões psíquicas. Identificou-se, assim, que a estratégia dialógica favoreceu a comunicação subjetiva de sentimentos e pensamentos em relação à estratégia tradicional.

A relação familiar e os aspectos econômicos também foram citados como problemas e dificuldades permanentes por alguns participantes do GT. Quanto ao convívio familiar, não houve diferença significativa ao longo do tempo (aumento de 1 ponto). No tocante ao financeiro, ocorreu uma redução de 3 pontos em relação ao pré-teste. Entre os participantes do GD houve melhora nos vínculos familiares (redução de 5 pontos) e no âmbito financeiro (redução de 9 pontos) durante o período pesquisado.

No tocante à relação familiar, os dados obtidos mostraram que os relacionamentos permaneceram prejudicados, apesar de o tratamento ter iniciado com redução do consumo, presença de sentimentos de arrependimento, além de apresentar resultados em relação ao início do processo de mudança.

\section{Discussão}

A melhor adesão verificada, ainda que discreta, dos participantes que se expuseram a estratégia dialógica, aplicada no GD, é aceita, pois permite maior aproximação do profissional com os relatos das histórias de vida. Isso decorre possivelmente do processo de identificação do próprio participante com as discussões construídas, planejadas e elaboradas com base nos contextos individuais, para o desenvolvimento de sua autonomia.

Estudos apontaram o vínculo como uma ferramenta que contribui para o estabelecimento da confiança entre profissionais e usuários assistidos devido a problemas por uso de substâncias psicoativas. Este é um recurso não invasivo, que possibilita a oferta do cuidado e o comprometimento dos profissionais, acarretando, como consequência, relações sociais saudáveis e livres de preconceitos $^{(12)}$.

No entanto, a possibilidade de outros fatores também interferirem precisa ser investigada $\mathrm{e}$ estudada em busca de melhorar o grau de envolvimento do usuário com o seu tratamento. Desse modo, assistir essa população torna-se um desafio diário, pois as relações estabelecidas pela pessoa que faz o uso prejudicial da substância apresentam específicas e complexas singularidades; são laços construídos entre o indivíduo e a droga, para o alívio de sintomas desconfortantes de sofrimento humano físicos e psíquicos ${ }^{(13)}$.

Já a estratégia educativa focada no modelo biomédico, tradicional, no qual a prescrição medicamentosa é o norteador do tratamento, tem como consequência o trabalho de uma equipe pouco motivada, apesar das ações e condutas da equipe multiprofissional. Logo, as frustrações surgem nos profissionais, pois não conseguem visualizar os efeitos concretos de seus esforços e de suas ações, sejam individuais, grupais ou em equipe ${ }^{(14)}$.

Posicionar aqueles que estão doentes como únicos responsáveis pelo sucesso do tratamento constitui-se atitude que afeta negativamente no caso de portadores de doenças crônicas, tornando-se um desafio para os profissionais ${ }^{(15)}$. A estratégia educativa aplicada pode refletir na dificuldade de adesão às propostas terapêuticas e de novos estilos de vida, conforme evidenciado no melhor resultado do GD em relação ao GT, no âmbito financeiro, social e familiar.

Uma das causas apontadas para a menor adesão dos participantes no GT foi o fato de que a estratégia tradicional pode ter uma efetividade mais baixa, quando se trata de facilitar a formação de vínculos entre usuários e entre usuários e profissionais nos espaços de saúde. Este é um fator bastante peculiar na adesão ao tratamento, já que os indivíduos possuem histórias de vínculos familiares conflituosos e fragilizados. Pesquisadores defendem que a construção de vínculos e a compreensão da relação dos usuários com as drogas são aspectos fundamentais para a adesão ao tratamento ${ }^{(16)}$. As situações enfrentadas e autorrelatadas neste estudo também foram encontradas em outras pesquisas, que identificaram, no relato dos participantes, alguns problemas no âmbito financeiro, social e da saúde, com maior reflexo nas questões nutricionais $^{(17)}$. 
As consequências do uso de drogas, independentemente de serem agudas ou crônicas, afetam a vida dos indivíduos e a de seus familiares e também a sociedade. Isso ocorre em curto, médio e longo prazos, uma vez que causam prejuízos em várias esferas da vida, como acidentes automobilísticos, gastos com internações hospitalares, doenças crônicas, infecções sexuais, o convívio social e familiar, bem como as atividades laborais. Tais aspectos afetam diretamente na QV dessas pessoas.

O fato de esses indivíduos e seus familiares buscarem a redução/interrupção do uso de drogas não exclui a expectativa e a oportunidade de recuperar a saúde física, mental e social, mediante ações educativas individuais ou coletivas. As pessoas buscam assistência em razão das graves sequelas ocasionadas ao organismo pela intoxicação, abstinência ou pelo consumo, como também pelas situações sociais e familiares construídas, marcadas por histórias de abandono, conflitos e estigmatização de modo singular ${ }^{(18)}$.

Neste estudo, supôs-se que a não redução dos problemas de saúde foi motivada pelo fato de as doenças apontadas no Quadro 1 estarem associadas ao consumo prolongado de drogas e, assim, foram cronificadas, a exemplo de gastrite e hipertensão. Além disso, outro fato observado foram as doenças psiquiátricas, neurológicas e agudas - como alucinações, transtornos convulsivos, depressão (Quadro 1). Estas requerem um tempo maior de tratamento e melhor suporte social, familiar, psicológico e financeiro. Este suporte, entretanto, é um aspecto difícil de ser conquistado pelos participantes deste estudo em um tempo tão curto ( 3 meses), apesar das tentativas para a mudança de hábitos e posturas.

Referida explanação também foi relatada por pessoas em tratamento em um município do Ceará, que reconheceram a associação de alguns desses agravos biopsicossociais decorrentes do uso de drogas, considerados por elas como algo muito desconfortante ${ }^{(19)}$. A busca pelo tratamento e as ações educativas favoreceram a descoberta e o diagnóstico de algumas doenças, como hanseníase, gastrite, hipertensão, diabetes que, pelas características, não obtêm estabilização ou cura em curto prazo.

Alterações irritativas diretas e durante a metabolização do álcool no organismo já foram comprovadas. Quando há associação com outros tipos de drogas, a probabilidade do surgimento e do agravamento de doenças instaladas de diversos tipos aumenta, a exemplo de doenças cardiopulmonares, gastrointestinais, hepáticas, neurológicas, psiquiátricas, hipotensão e até a morte $^{(20)}$. Além disso, há ainda o desgaste dos vínculos ocasionados pelas situações decorrentes da dependência química ${ }^{(21)}$.

Em relação aos problemas psíquicos mais referidos no GD (ver Quadro 1), outros estudos também verificaram que usuários em tratamento possuíam problemas como depressão, tipo moderado a grave, ansiedade e estresse ${ }^{(22-23)}$.

No âmbito da saúde, no que se refere a não haver melhora nas queixas referidas por esses indivíduos, é compreendida, uma vez que esses vivenciam dificuldades em vários aspectos de sua vida. Essas podem ser de ordem psicossocial, familiar, econômica e cultural, e influenciam suas percepções e contextos em relação à sua qualidade de vida.

Nesse sentido, neste estudo, os participantes reconheceram que os problemas decorriam do consumo descontrolado de drogas, e afetavam negativamente sua qualidade de vida e a de seus familiares. Estudo avalia que as situações vivenciadas pelos familiares, sobretudo no âmbito emocional, causam danos irreparáveis ${ }^{(24)}$. Por isso, torna-se difícil a superação dos traumas ocasionados. Em contrapartida, a família é parte fundamental, para que ocorra o equilíbrio e o recomeço dessas pessoas para o enfrentamento da doença.

Resultados contrários foram encontrados em outro estudo $^{(25)}$, ao ser aplicado um questionário sobre a percepção da QV dos participantes. Estes apresentaram percepções positivas: 57\% dos entrevistados avaliaram como boa e 39\% acreditavam que sua QV era semelhante à de outras pessoas, não estando associada à doença. Entretanto, esses participantes relataram boas condições sociais, econômicas e familiares, o que 
não ocorreu com os participantes desta pesquisa. A identificação dessa discordância é aceita porque, no momento da entrevista realizada, os participantes informaram boas condições de saúde e relacionamentos familiares satisfatórios, além de que $72 \%$ da amostra estavam empregados, dado que pode ter afetado decididamente no resultado da pesquisa ${ }^{25}$.

Diante do exposto, fica evidente que os profissionais atuantes na assistência devem promover reflexões para resgatar a autoestima, autonomia e reinserção social dessas pessoas. São histórias de vida conflitantes e de perdas nos diversos âmbitos, cujo tratamento pode tornar-se o único recurso ${ }^{(14)}$ para promover melhor $\mathrm{QV}$ e incentivar a busca de nova trajetória, para iniciar uma nova história.

Apesar de haver concordância no tocante à importância de ações profissionais para o sucesso do tratamento, uma das limitações deste estudo foi a falta de pesquisas anteriores, que relacionassem o impacto da educação em saúde nos diversos âmbitos da vida dessas pessoas em tratamento por uso prejudicial de substâncias psicoativas, com abordagem quantitativa. Outra limitação consistiu na realização desta pesquisa em apenas uma unidade de saúde e na utilização de uma amostragem por conveniência.

\section{Conclusão}

A estratégia educativa dialógica, desenvolvida em grupos e individualmente, quando comparada à estratégia educativa tradicional, demonstrou ser mais efetiva para melhorar a adesão do participante ao tratamento, as relações familiares, como também para identificar estados emocionais e psíquicos. Isso ocorreu pelo fato de essa estratégia possibilitar maior aproximação entre o profissional e os indivíduos em tratamento e possibilitar o diálogo com o contexto de vida, os quais interferem na tomada de decisão, no desejo de mudança e na mudança de hábitos, influenciando diretamente na QV.

No entanto, é preciso que sejam realizados mais estudos envolvendo os impactos das ações profissionais nos contextos dos indivíduos assistidos, e que possam ser aplicados em diferentes realidades por maior tempo, a fim de que os problemas vivenciados pelos usuários que buscam o tratamento sejam diminuídos por meio da assistência profissional baseada em evidências científicas.

\section{Colaborações:}

1 - concepção, projeto, análise e interpretação dos dados: Cláudia Cristina Rolim da Silva e Célio Fernando de Sousa Rodrigues;

2 - redação do artigo e revisão crítica relevante do conteúdo intelectual: Cláudia Cristina Rolim da Silva e Célio Fernando de Sousa Rodrigues;

3 - aprovação final da versão a ser publicada: Cláudia Cristina Rolim da Silva e Célio Fernando de Sousa Rodrigues.

\section{Agradecimentos}

Aos participantes da pesquisa, profissionais e pesquisadores de saúde mental.

\section{Referências}

1. Seidl EMF, Zannon CMLC. Qualidade de vida e saúde: aspectos conceituais e metodológicos. Cad Saúde Pública. 2004 abr;20(2):580-8. DOI: https://doi.org/10.1590/S0102-311X2004000200027

2. Queiroz FA, Pace AE, Santos CB. Adaptação Cultural e Validação do Instrumento Diabetes -39 (D-39): Versão para Brasileiros com Diabetes Mellitus Tipo 2 - Fase 1. Rev Latino-Am Enfermagem. 2009 out;17(5):708-15. DOI: http://dx.doi.org/10.1590/ S0104-11692009000500018

3. Damasceno RO, Boery RNSO, Ribeiro IJS, Anjos KF, Santos VC, Boery EN. Uso de álcool, tabaco e outras drogas e qualidade de vida de estudantes universitários. Rev baiana enferm. 2016;30(3):1-10. DOI: http://dx.doi.org/10.18471/ rbe.v30i3.15533

4. Morais M, Paixão R. Significados e vivências em torno da adição de substâncias: teoria fundamentada nos dados. Rev baiana enferm. 2020;34:e35002. DOI: http://dx.doi.org/10.18471/ rbe.v34.35002 
5. Kirchner MM, Pimentel RRS, Rodrigues TFCS, Radovanovic CAT, Scardoelli MGC. Recaídas e a busca pelo tratamento de usuários de crack. Rev enferm UFPE on line. 2020;14:e243627. DOI: https://doi.org/10.5205/1981-8963.2020.243627

6. Brasil. Ministério da Saúde. Secretaria de Gestão Estratégica e Participativa. Departamento de Apoio à Gestão Participativa. Caderno de educação popular e saúde [Internet]. Brasília (DF); 2007 [cited 2019 Nov 11]. Available from: http://bvsms.saude.gov.br/bvs/publicacoes/ caderno_educacao_popular_saude_p1.pdf

7. Brasil. Ministério da Educação. Diretrizes Curriculares Nacionais do Curso de Graduação em Enfermagem [Internet]. Brasília (DF); 2001 [cited 2018 May 13]. Available from: http://portal. mec.gov.br/cne/arquivos/pdf/Enf.pdf

8. Figueiredo MFS, Rodrigues-Neto JF, Leite MTS. Modelos aplicados às atividades de educação em saúde. Rev Bras Enferm. 2010 jan-fev;63(1):117-21. DOI: http://dx.doi.org/10. 1590/S0034-71672010000100019

9. Freire P. Pedagogia do oprimido. 17a ed. Rio de Janeiro: Paz e Terra; 1987.

10. Silva JV, Machado DR, Martins JCC, Narcy JL, Porfirio TA, Andrade FN. Capacidades de autocuidado e sua relação com os fatores condicionantes básicos: um estudo em Unidades Básicas de Saúde. Rev Eletrônica Acervo Saúde. 2012;4(1):185-99. DOI: http://dx.doi.org/10.1590/ S0080-623420130000500020

11. Brasília. Secretaria Nacional de Políticas sobre Drogas. Sistema para detecção do uso abusivo e dependência de substâncias psicoativas: encaminhamento, intervenção breve, reinserção social e acompanhamento [Internet]. 7a ed. Brasília (DF); 2014. (SUPERA). [cited 2018 Mar 21]. Available from: https://www.supera.org.br/@/ material/mtd/pdf/SUP/SUP_Mod3.pdf

12. Bittencourt MN, Pantoja PVN, Silva Jr PCB, Pena JLC, Nemer CRB, Moreira RP. Street clinic: the care practices with users of alcohol and other drugs in Macapá. Esc Anna Nery. 2019.23(1):e20180261. DOI: http://dx.doi.org/10. 1590/2177-9465-ean-2018-0261

13. Bica SCL, Oliveira MM, Cruz VD. A pedra é o meu remédio: usuários de crack na percepção da própria saúde. SMAD. Rev Eletrônica Saúde Mental Álcool Drog. 2019;15(1):50-6. DOI: http://dx.doi.org/10.11606/issn.1806-6976. smad.2019.151787
14. Kolhs M, Olschowsky A, Ferraz L. Sofrimentos e defesas no trabalho em um serviço de saúde mental. Rev Bras Enferm. 2019 Aug;72(4):903-9. DOI: http://dx.doi.org/10.1590/0034-7167-2018-0140

15. Gusmão JL, Mion Jr D. Adesão ao tratamento conceitos. Rev Bras Hipertens [Internet]. 2006 [cited 2020 Aug 5];13(1):23-5. Available from: http://departamentos.cardiol.br/dha/revista/13-1/ 06-adesao-ao-tratamento.pdf

16. Ferreira ISS, Souza SL. Adesão ao Tratamento de Usuários de um CAPS AD do Interior da Bahia. Trabalho apresentado no Seminário de Iniciação Científica. Feira de Santana (BA); 2018. Anais (on-line). Feira de Santana; 2018. DOI: http:// dx.doi.org/10.13102/semic.v0i21.3728

17. Andrade SP, Lima CR, Orange LG, Medeiros TB, Santos REA, Santos HCM, et al. Estado nutricional de pacientes alcoolistas de uma instituição hospitalar do Nordeste brasileiro. Nutr clín diet Hosp. 2016;36(2):63-73. DOI: http://dx.doi.org/10.12873/362pereiraandrade

18. Machado AR, Modena CM, Luz ZMP. O que pessoas que usam drogas buscam em serviços de saúde? Compreensões para além da abstinência. Interface (Botucatu). 2020;24:e190090. DOI: https://doi.org/10.1590/interface.190090

19. Frota GAS, Martins KMC, Dourado JVL, Aguiar FAR, Gurgel Júnior FF. Experiência de usuários acerca do uso de drogas. Rev Bras Promoç Saúde. 2018;31(3):1-11. DOI: https://doi. org/10.5020/18061230.2018.7868

20. Pontes MA. Transtornos de abuso de drogas. In: Springhouse. Enfermagem psiquiátrica. 3a ed. São Paulo: Guanabara Koogan; 2006. Série Incrivelmente Fácil. cap. 11.

21. Cavalcante LP, Falcão RST, Lima HP, Marinho AM, Macedo JQ, Braga VAB. Rede de Apoio Social ao Dependente Químico: Ecomapa como instrumental na assistência em saúde. Rev Rene [Internet]. 2012 [cited 2019 Aug 15];13(2):321-31. Available from: http:// repositorio.ufc.br/bitstream/riufc/4408/1/2012_ art_jqmacedo.pdf

22. Lemes AG, Nascimento VF, Rocha EM, Almeida MASO, Volpato RJ, Luis MV. Terapia Comunitária como cuidado complementar a usuários de drogas e suas contribuições sobre a ansiedade e a depressão. Esc Anna Nery 2020. 24(3):e20190321. DOI: http://dx.doi. org/10.1590/2177-9465-ean-2019-0321 
23. Andretta I, Limberger J, Jaluza A, Mello LTN. Sintomas de depressão, ansiedade e estresse em usuários de drogas em tratamento em comunidades terapêuticas. Psico-USF. 2018;23(2):361-73. DOI: https://dx.doi.org/10.1590/1413-827120182 30214

24. Cardoso MP, Dall Agnol R, Taccolini C, Tansini K, Vieira A, Hirdes A. A percepção dos usuários sobre a abordagem de álcool e outras drogas na atenção primária à saúde. Aletheia [Internet]. $2014 \mathrm{dez}$ [cited 2019 Aug 11];(45):72-86. Available from: http:// pepsic.bvsalud.org/scielo.php?script=sci arttext\&pid=S1413-03942014000200006\&lng=pt

25. Silveira GL, Rodrigues LB. O consumo de substâncias psicoativas e o autocuidado entre pessoas em situação de rua na cidade de Juazeiro - BA. Rev Psicol Diversidade Saúde. 2013 abr;1(1):95-122. DOI: http://dx.doi.org/ 10.17267/2317-3394rpds.v2i1.212

Recebido: 28 de setembro de 2020

Aprovado: 1 de fevereiro de 2021

Publicado: 5 de abril de 2021

A Revista Baiana de Enfermagem utiliza a Licença Creative Commons - Atribuição-NãoComercial 4.0 Internacional. https://creativecommons.org/licenses/by-nc/4.0/

Este artigo é de acesso aberto distribuído sob os termos da Licença Creative Commons (CC BY-NC). Esta licença permite que outros remixem, adaptem e criem a partir do seu trabalho para fins não comerciais. Embora os novos trabalhos tenham de lhe atribuir o devido crédito e não possam ser usados para fins comerciais, os usuários não têm de licenciar esses trabalhos derivados sob os mesmos termos. 